\title{
True umbilical cord knot and tight nuchal cord loops as a cause of fetal distress and emergent
}

\section{cesarean delivery: A Case report}

\section{Meral Rexhepi*}

International Balkan University, Skopje, North Macedonia

*Corresponding Author: Meral Rexhepi, International Balkan University, Skopje, North Macedonia

Received date: 30 November 2021; Accepted date: 10 December 2021; Published date: 18 December 2021

Citation: Rexhepi M (2021) True umbilical cord knot and tight nuchal cord loops as a cause of fetal distress and emergent cesarean delivery: A Case report. J Med Case Rep Case Series 2(18): https://doi.org/10.38207/JMCRCS/2021/0218250

Copyright: (C) 2021 Meral Rexhepi. This is an open-access article distributed under the terms of the Creative Commons Attribution License, which permits unrestricted use, distribution, and reproduction in any medium, provided the original author and source are credited.

\begin{abstract}
A nuchal cord (NC) is a complication that occurs when the umbilical cord wraps around the fetal neck one or more times. True knots (TK) of the umbilical cord are complications that can result in perinatal adverse outcomes on the infant, including fetal asphyxia and fetal death. We report a very uncommon case of triple NC coexisting with a $\mathrm{TK}$ of the umbilical cord in a 23 -year-old primigravida, who was admitted at 40 weeks gestation, with labor pains, with a normal sonographic examination and cardiotocography profile at the beginning of labor. During the initial stage of labor, the fetus began to have recurrent deep atypical variable decelerations with a progressive reduction in the variability and prolonged bradycardia. Five hours after active phasis of labor, due to this repetitive situation of fetal bradycardia and decelerations, it was decided to perform an emergent caesarean section. A live female neonate was born, weighing 2760 g with Apgar scores of 7 and 9 at 1 and 5 minutes, respectively. Our case described that a loop of the umbilical cord around the fetal neck can cause variable decelerations in antenatal fetal heart monitoring. The NC in the presence of the true umbilical knot is associated with an increased risk of fetal distress compared with either of these entities alone. There is a need for routine continuous monitoring of patients in labor using cardiotocography.
\end{abstract}

Keywords: Nuchal cord, True umbilical knot, Fetal distress

\section{Introduction}

The umbilical cord (UC) is the anatomic tubular structure that physically connects the developing intrauterine fetus to the placenta, which is anchored to the maternal uterine wall [1]. Long UC are present in $4 \%$ of placentas and pose a higher risk for cord entanglement (i.e., true knots or cord loops around the neck or body parts), circulatory stasis, and vascular thrombosis [2]. Nuchal cord (NC) has been defined as a $>360$ wrapping of the umbilical cord around the fetal neck [3]. NC occurs in about 10-29\% of fetuses and the incidence increases with advancing gestation age. Most are not associated with perinatal morbidity and mortality, but a few studies have shown that $\mathrm{NC}$ can affect the outcome of delivery with possible long-term effects on the infants [4]. The incidence of single, double, triple, or quadruple $\mathrm{NC}$ at delivery has been reported as $10.6 \%, 2.5$ $\%, 0.5 \%$, and $0.1 \%$, respectively [5]. Acute umbilical disorders (by

\section{Case report}

We describe a case of acute intrapartum fetal hypoxia, with the extraction of the fetus by an emergency c-section and finding of a true umbilical cord knot and three tight nuchal cord loops around the fetal neck. The patient was 25 years old primigravida (G1P0). She has admitted at 40 weeks of pregnancy with labor pains. She had regular antenatal checkups and the antenatal period was uneventful. The course of pregnancy was without complications. The growth scan performed at the time of hospitalization, showed normal growth of compression, knot, or prolapse) are relatively common obstetric events that could significantly affect perinatal morbidity. The reported incidence of true umbilical cord knots (CK) is $1.2 \%$ for all pregnancies [6]. and certain factors have been noted to increase its predisposition. These include long umbilical cords, polyhydramnios, small size fetuses, male fetuses, gestational diabetes mellitus, monoamniotic twins, the process of undergoing genetic amniocentesis, and multiparity [7]. In contrast to single NC, which occur in $15.8-30 \%$ of singleton fetuses at term and have not been unequivocally associated with significant adverse perinatal outcome, TK of the umbilical cord occur in $0.04-3 \%$ of deliveries and have been associated with perinatal morbidity in $11 \%$ of cases and a 4-10fold increase in the risk of stillbirth [8].

the fetus with a normal volume of amniotic fluid, with a grade three maturity placenta inserted on the anterior uterine wall. Her antenatal blood workup and ultrasound were within normal limits. She had no history of hypertensive disorders, gestational diabetes, or thyroid disorders. At the 12th week of gestation, she underwent a firsttrimester screening (nuchal translucency + first-trimester serum markers) and was found to have no risk for chromosomal defects. At admission, an obstetric clinical examination showed that the breech 
end of the fetus was presenting, with the fetal back to the right and the small parts to the left. A sonographic examination using 2dimensional imaging was normal, including growth and the biophysical profile. Doppler sonography revealed a normal umbilical artery waveform with no notching or evidence of cord compression.

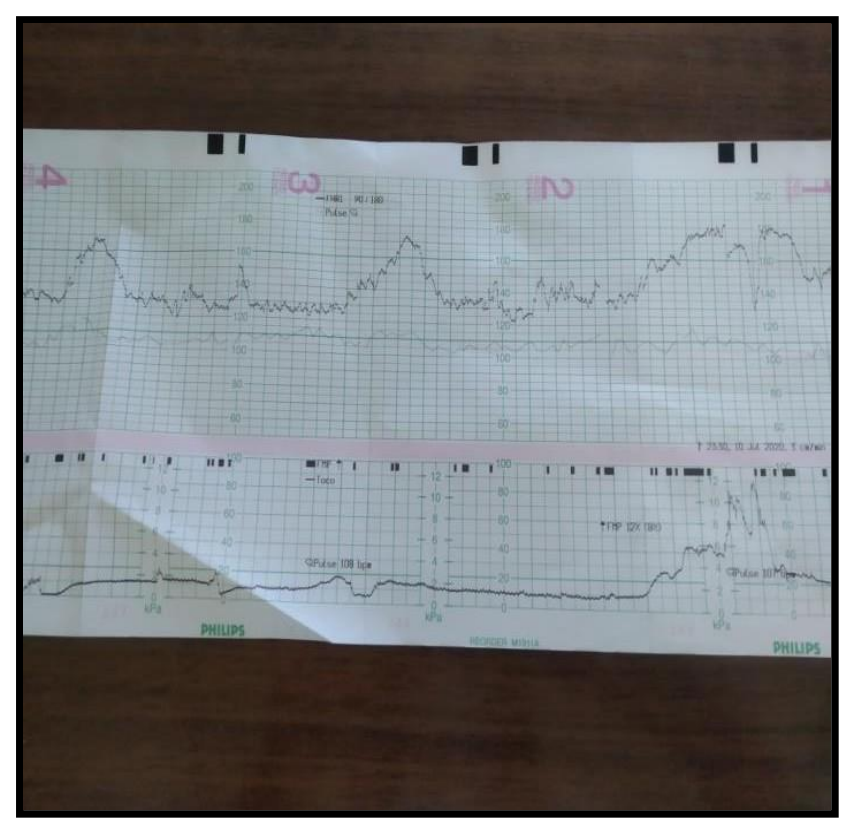

Figure 1: Reactive NST in the beginning

Figure 2: Deep decelerations after 5 of labor hour of initial stage of labor

The situation did not improve on changing the patient's position. Due to this repetitive situation of fetal bradycardia and decelerations, it was decided to perform an emergent caesarean section in spinal anesthesia. A live female neonate was born at 40 weeks 1 day, weighing $2760 \mathrm{~g}$ with Apgar scores of 7 and 9 at 1 and 5 minutes, respectively. There was no excess liquor or meconium staining and

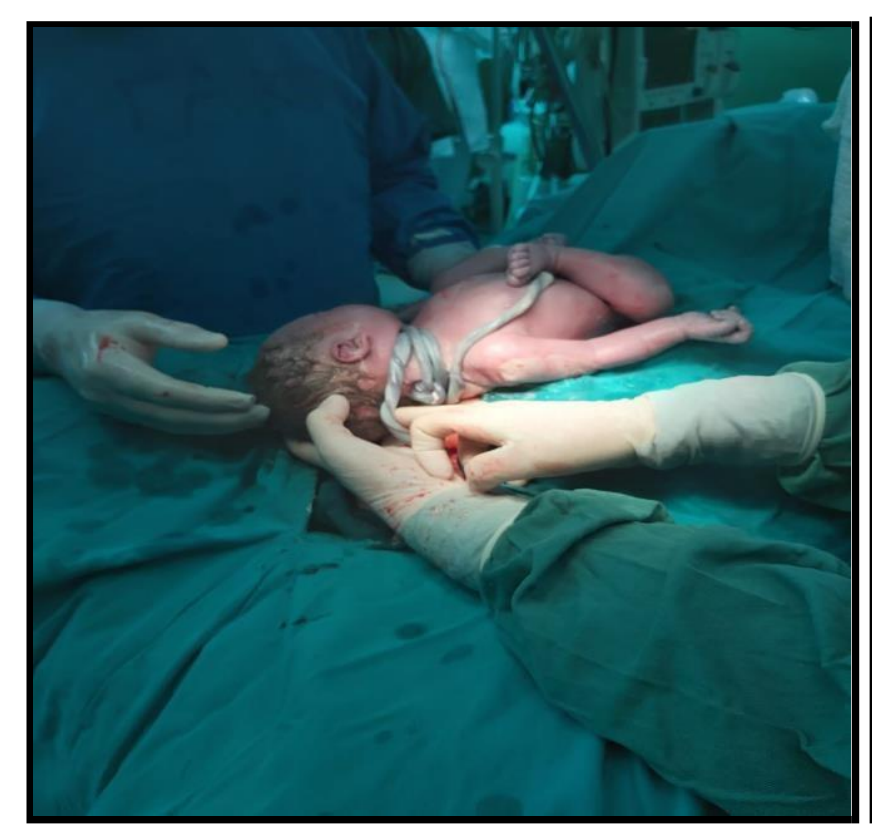

Figure 3: Triple nuchal cord and true knot

Figure 4: A true knot after removal knot of the nuchal cord

\section{Discussion}

True knot at delivery is rare and is not associated with repetitive late decelerations, other non-reassuring electronic fetal monitoring parameters, or neonatal morbidity. The presence of a true knot at delivery is clinically benign and should not be a cause for concern [9]. Nuchal cords can cause cord compression, leading to obstruction of blood flow in the thin-walled umbilical vein, while blood continues to be pumped out through the thicker-walled umbilical arteries causing hypovolemia, hypotension, and fetal hypoxia [10]. The
At the beginning of labor, the non-stress evaluation showed a normal profile. (Figure 1) During the initial stage of labor (with the cervix dilated $5 \mathrm{~cm}$ and at a DeLee fetal station of -2 ), the fetus began to have recurrent deep atypical variable decelerations with a progressive reduction in the variability and prolonged bradycardia. (Figure 2).

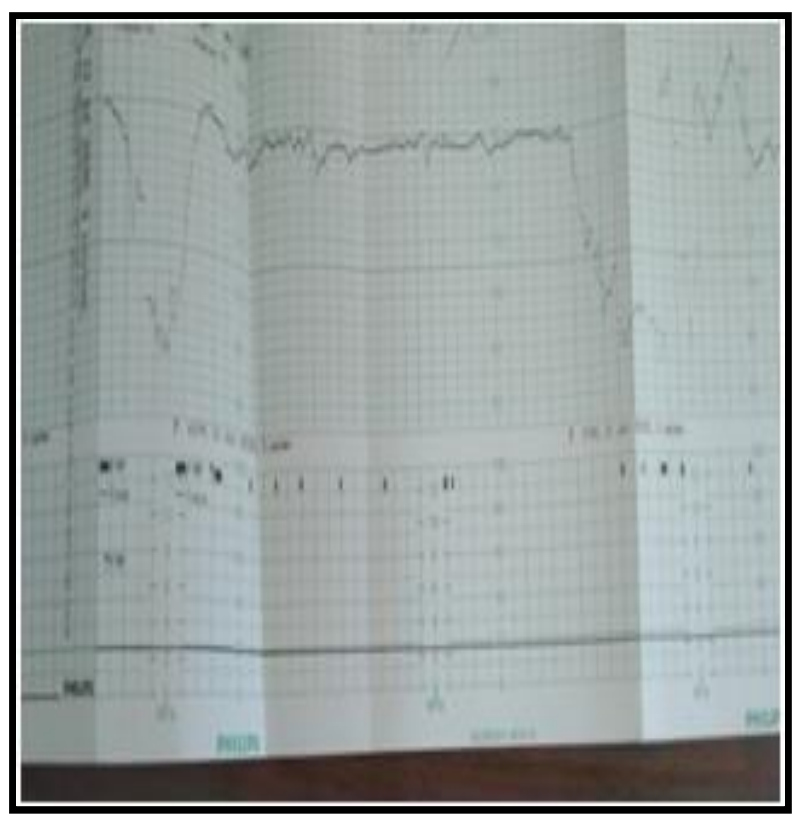

blood loss was minimal. A true knot and triple nuchal cord were confirmed at birth. (Figure 3 and 4). Examination of the placenta showed an excessively long umbilical cord measuring $124 \mathrm{~cm}$ with one loose true knot. The placenta weighed $475 \mathrm{~g}$ and morphologically was normal.

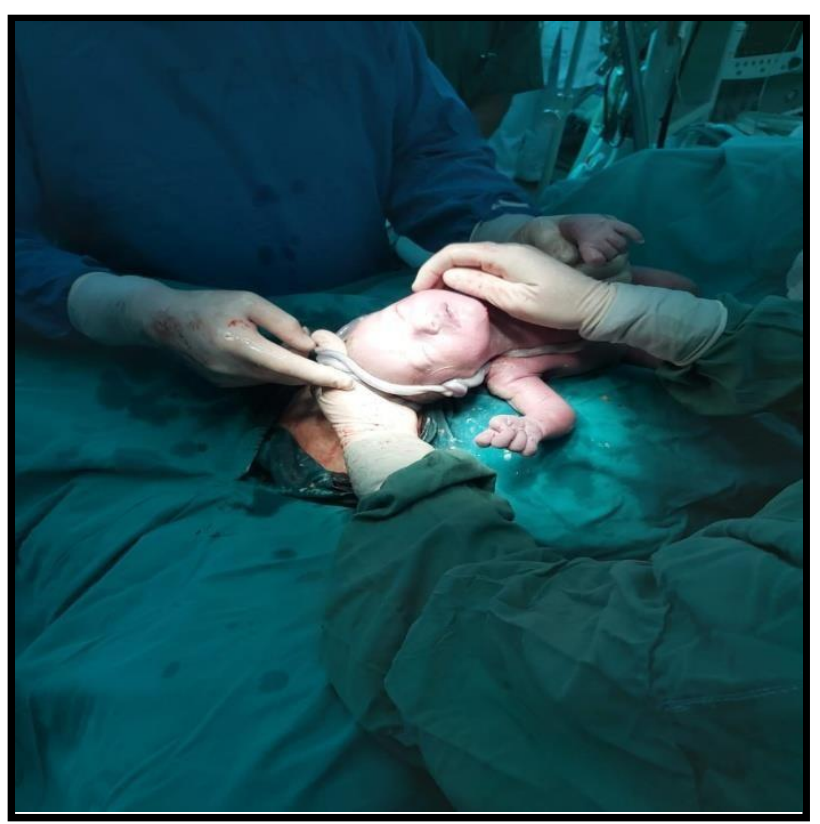

nuchal cord can be single or multiple and tight or loose. During labor, the only indication of a nuchal cord or a cord being around the baby's body may be variable fetal heart decelerations more likely seen with uterine contractions as this is the time the cord gets stretched more tightly. Intrapartum complications like fetal heart rate irregularities and meconium staining of amniotic fluid are thus increased in the nuchal cord group [11]. Single and multiple nuchal cords, and true $\operatorname{knot}(\mathrm{s})$ of the umbilical cord, are often incidental findings noted at 
delivery of non-hypoxic non-acidotic newborns without any evidence of subsequent adverse neonatal outcome [12]. A prospective cohort study of 8,580 singleton pregnancies, which aimed to determine the association between nuchal cord, electronic fetal monitoring parameters, and adverse neonatal outcomes, showed that the nuchal cord was associated with category II electronic fetal monitoring parameters, which may drive increased rates of operative vaginal delivery, but there was no significant association with neonatal morbidity [13]. The retrospective study of 4,404 singleton term pregnancies which aimed to compare the fetal outcomes and mode of delivery in patients with nuchal cord for a single loop, double loops, and multiple loops showed that the only nuchal cord for 3 turns or more was associated with a higher incidence of suspected fetal distress. However, the Apgar scores were not affected. Therefore, a nuchal cord of any turns was not associated with adverse fetal outcomes [14].

The presence of knots during the third trimester and labor does not seem to be associated with increased perinatal and intrapartum morbidity and mortality, although there is still some controversy in the literature on this topic [15]. Raisanen et al. showed that the true umbilical cord knot is relatively common and is associated with increased incidence of SGA infants, premature birth, need for neonatal intensive care, and fetal death [16]. The process of delivering these babies is precarious, and in case of fetal distress and severe hypoxia of the fetus, it can evolve from a normal delivery to a high-

\section{Conclusions}

A true knot of the umbilical cord in the presence of a coexisting nuchal cord may be associated with an increased risk of adverse perinatal outcomes compared with either of these entities alone. The neonate was delivered by emergent c-section following spontaneous

\section{References}

1. Fahmy M (2018) Anatomy of the Umbilical Cord. In: Umbilicus and Umbilical Cord. Springer, Cham.

2. Zbeidy R, Souki FG (2017) One long umbilical cord, four nuchal cord loops and a true knot. BMJ Case Rep. 2017: bcr2017223241.

3. Henry E, Andres RL, Christensen RD (2013) Neonatal outcomes following a tight nuchal cord. Journal of Perinatology. 33(3): $231-234$

4. Gutvirtz G, Wainstock T, Masad R, Landau D, Sheiner E (2019) Does nuchal cord at birth increase the risk for cerebral palsy? Early Hum Dev. 133: 1-4.

5. Sherer DM, Dalloul M, Sabir S, London V, Haughton M, et al. (2017) Persistent quadruple nuchal cord throughout the third trimester associated with decelerating fetal growth. Ultrasound Obstet Gynecol. 49(3): 409-410.

6. Noval BD, Llaneza IB, Sepúlveda MR, Ferrer FJ, Carmen FB (2019) True umbilical cord knot, an emergency during labor. Clin Case Rep. 7(11): 2242-2244. risk emergent cesarean section [17]. In our case necessitating cesarean delivery for prolonged fetal bradycardia and deep decelerations suggests a possible cumulative increased risk of adverse perinatal outcome in the presence of coexisting true knot of the umbilical cord and multiple nuchal cords. A recent study showed that the UC abnormalities and mostly multiple UC entanglements are associated with the development of non-reassuring fetal heart rate and adverse neonatal outcomes. According to the study by Weiner et al, which analyzed the UC anomalies in two comparative groups showed that UC entanglements, true knots, and short cords were all more common in the emergency cesarean delivery group compared with the vaginal delivery group. [18] The pregnancy presented in our report showed not any of the antenatal risk factors of CK, such as multiparity, prolonged pregnancy, genetic amniocentesis, previous spontaneous abortion, a male fetus, polyhydramnios, or reversible arterial redistribution in the fetus. We think that the only reason for the triple nuchal cord and the true knot is the very long NC. The combined effect of entanglement and knot on the risk of intrauterine or perinatal death is more than additive [19]. Considering the relatively high incidence of single nuchal cords at term and the relatively infrequent observation of a true knot of the umbilical cord (often noted incidentally at delivery), guidelines have not yet been provided for the management of pregnancies complicated by prenatal diagnosis of nuchal or true knots of the umbilical cord [6].

labor at 40 weeks of gestation due to concern for fetal demise. Routine continuous monitoring of patients in labor using cardiotocography is the best way to achieve a good outcome.

7. Ikechebelu JI, Eleje GU, Ofojebe CJ (2014) True Umbilical Cord Knot Leading to Fetal Demise. Ann Med Health Sci Res. 4(Suppl 2): S155-S158.

8. Sherer DM, Dalloul M, Ward K, Nakagawa J, Joseph I, et al. (2017) Coexisting true umbilical cord knot and nuchal cord: possible cumulative increased risk of adverse perinatal outcome. Ultrasound Obstet Gynecol. 50(3): 404-405.

9. Carter EB, Chu CS, Thompson Z, Tuuli MG, Macones GA, et al. (2018) True knot at the time of delivery: electronic fetal monitoring characteristics and neonatal outcomes. J Perinatol. 38(12): 1620-1624.

10. Peesay M (2017) Nuchal cord and its implications. Matern Health Neonatol Perinatol. 3: 28

11. Tayade S, Kore J, Tayade A, Gangane N, Thool K, et al. (2018) Is Nuchal cord a cause of concern? Madridge J Womens Health Emancipation. 2(1): 46-50. 
12. Sherer DM, Amoabeng O, Dryer AM, Dalloul M (2020) Current Perspectives of Prenatal Sonographic Diagnosis and Clinical Management Challenges of True Knot of the Umbilical Cord. Int J Womens Health. 12: 221-233.

13. Carter EB, Chu CS, Thompson Z, Tuuli MG, Macones GA, et al. (2020) Electronic Fetal Monitoring and Neonatal Outcomes when a Nuchal Cord Is Present at Delivery. Am J Perinatol. 37(4): 378383.

14. Kong CW, Chan LW, To WWK (2015) Neonatal outcome and mode of delivery in the presence of nuchal cord loops: implications on patient counselling and the mode of delivery. Arch Gynecol Obstet. 292(2): 283-289.

15. Bakas P, Papadakis E, Hassiakos D, Liapis A (2013) Secondtrimester miscarriage and umbilical cord knot. Case report and review of the literature. Clin Exp Obstet Gynecol. 40(3): 448-451.

16
Journal of Medical Case Reports and Case Series ${ }^{2}$ ISSN: 2692-9880 Räisänen S, Georgiadis L, Harju M, Keski-Nisula L, Heinonen S

(2013) True umbilical cord knot and obstetric outcome. Int J Gynaecol Obstet. 122(1): 18-21.

17. Haghighi L, Jahanshahi F, Dini P (2020) Two knots in an umbilical cord with seventy-centimeter length: A case report. Clin Case Rep. 8(8): 1579-1581.

18. Weiner E, Fainstein N, Schreiber L, Sagiv R, Bar J, et al. (2015) The association between umbilical cord abnormalities and the development of non-reassuring fetal heart rate leading to emergent cesarean deliveries. J Perinatol. 35(11): 919-923.

19. Linde LE, Rasmussen S, Kessler J, Ebbing C (2018) Extreme umbilical cord lengths, cord knot and entanglement: Risk factors and risk of adverse outcomes, a population-based study. PLoS ONE. 13(3): e0194814. 